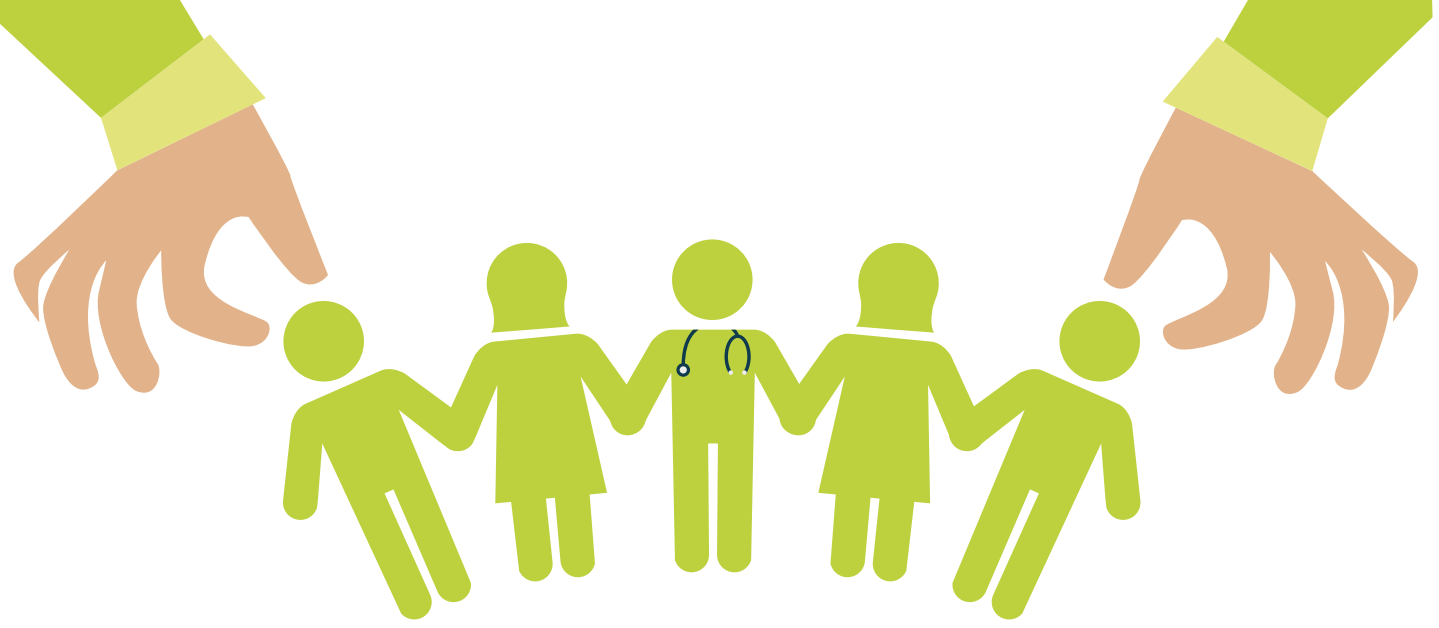

\title{
SISTEMAS DE INFORMAÇÃO COMO APOIO À GESTÃO DE RECURSOS HUMANOS EM SAÚDE
}

\author{
INFORMATION SYSTEM AS SUPPORT OF HUMAN RESOURCES MANAGEMENT ON HEALTH
}

\author{
Giveldna Maria Costa Pereira \\ Universidade Federal do Rio Grande do Norte, Centro de Tecnologia, Campus Universitário, Lagoa Nova, \\ Natal, RN, Brasil. CEP: 59072-970. giveldnap@gmail.com \\ Idelmárcia Dantas de Oliveira \\ Instituto Federal de Educação, Ciência e Tecnologia do Rio Grande do Norte, Campus Ceará-Mirim, Av. \\ Planalto, Km 406, Planalto, Ceará-Mirim, RN, Brasil, CEP: 59.580-000.Idelmacria.dantas@ifrn.edu.br \\ Rodrigo Dantas da Silva \\ Graduando em Engenharia de Computação e Automação pela Universidade Federal do Rio Grande do \\ Norte, pesquisador do Laboratório de Inovação Tecnológica em Saúde \\ Hélio Roberto Hékis \\ Universidade Federal do Rio Grande do Norte, Departamento de Engenharia de Produção, Campus \\ Universitário, Lagoa Nova, Natal, RN, Brasil. CEP: 59072-970. hekis1963@gmail.com \\ Ricardo Alexsandro de Medeiros Valentim \\ Universidade Federal do Rio Grande do Norte, Departamento de Engenharia Biomédica, Campus \\ Universitário, Lagoa Nova, Natal, RN, Brasil. CEP: 59072-970. ricardo.valentim@ufrnet.br
}

\section{RESUMO}

Este artigo apresenta uma revisão na literatura, nacional e internacional, entre 2005 e 2015, sobre o tema "Sistemas de informação que apoiam à gestão de recursos humanos em saúde", tendo como objetivo principal identificar quais sistemas de informação foram desenvolvidos para apoiar a gestão de recursos humanos em saúde. A metodologia adotada consistiu em duas etapas: Revisão bibliográfica e Análise detalhada do conteúdo. A pesquisa desenvolvida foi classificada como bibliográfica, exploratória e quantitativa. Os principais resultados desta pesquisa foram: $82,35 \%$ dos trabalhos são artigos científicos, publicados em 2009, 2010 e 2013, pela USP, UFPE e Universidade de Washington, na área de Ciências da Saúde; 74,02\% dos estudos possuem abordagem qualitativa, $80,39 \%$ são do tipo bibliográficos e apresentam como principais focos de interesse a avaliação e explanação sobre a importância de sistemas de informação; a grande maioria dos sistemas identificados estão em produção, apresentando a estratégia de desenvolvimento Web e somente dois deles foram desenvolvidos para apoiar a gestão de recursos humanos em saúde. Por fim, foi proposta e apresentada a Plataforma RH do SUS, em desenvolvimento pelo LAIS/UFRN.

PALAVRAS-CHAVE: Sistema de informação, Recursos humanos, Gestão de recursos humanos, Saúde.

\section{ABSTRACT}

This article presents a review based on national and international literature, between 2005 and 2015, about "Information system that support the human resources management on health ". Having as main objective to identify which information system has been 
developed to support the human resources management on health. The adopted methodology consisted in two phases: Bibliographic review and the detailed analysis about the content. The developed research has been classified as bibliographic, exploratory and quantitative. The main results of this research has been: $82,35 \%$ of the researches are scientific articles, published on 2009, 2010 and 2013, by USP, UFPE and University of Washington, at the Health and Science area; $74,02 \%$ of these studies has qualitative approach, $80,39 \%$ are bibliographic and focus on evaluation and explanation about the matter of information system; Most of the identified systems are on production, showing the strategy of web development and only two of them has been made to support the management of human resources on health. Lastly, we have proposed and presented the HR Platform of SUS, in development by LAIS/UFRN.

KEYWORDS: Information system, resources human, human resources management, health.

\section{INTRODUÇÃO}

A evolução tecnológica da informação tem impulsionado, cada vez mais, os gestores de diversos segmentos a considerar o uso de sistemas de informação para gerenciar informações que serão utilizadas no processo de tomada de decisão. Tratando-se de decisões que envolvem um direito básico e constitucional, como é o caso da saúde, é ainda mais evidente a importância da informação, desde que completa, correta e oportuna. Por exemplo, para definir o número de médicos que deverão atuar em determinada região do país é necessário conhecer o número de unidades de saúde por região, o número de pacientes atendidos por unidade, dentre outros. De outra forma, a distribuição de recursos não atenderá as reais necessidades de um país com dimensões continentais como o nosso. Além disso, a informação também é essencial para a gestão da saúde, para a descentralização das atividades e para promover o controle social, que pode ser efetivado pela sua divulgação em portais públicos, mantidos pelo Ministério da Saúde.
Um Sistema de Informação em Saúde (SIS), em consonância com a definição do Ministério da Saúde, pode ser compreendido como um mecanismo de coleta, de processamento e de análise das informações necessárias para o planejamento dos serviços de saúde. Assim, os sistemas de informações direcionados a atenção em saúde podem atuar como ferramenta importante para adquirir, organizar e analisar dados necessários à definição de problemas e riscos para a saúde, além de serem importantes para avaliar a eficácia, a eficiência e influência que os serviços prestados possam ter no estado da saúde populacional e, sobretudo, contribuir para a produção de conhecimento em saúde ${ }^{1}$.

O Brasil dispõe de uma ampla rede de Sistemas de Informação em Saúde, estando grande parte de suas informações disponíveis na Internet, via Departamento de Informática do Sistema Único de Saúde (DATASUS). Acompanhando o processo de evolução dos Sistemas de Informação que atuam nos ambientes empresariais, na saúde também há uma variedade de sistemas desenvolvidos para atender dimensões isoladas, tais como: Sistema de informações sobre nascidos vivos (Sinasc); Sistema de informação sobre agravos de notificação (Sinan); Sistema de informações hospitalares $(\mathrm{SIH})$, Sistema de informação de mortalidade (SIM), dentre outros. Os dados desses e de outros sistemas são, então, reunidos e difundidos pelo DATASUS e pela Rede Interagencial de Informática para a Saúde (Ripsa), responsáveis por difundir, de forma organizada, dados básicos das condições de saúde ${ }^{2}$.

Os dados supracitados são capazes de compor uma grande gama de indicadores e retratam as condições administrativas dos serviços ${ }^{8}$. Porém, as informações sobre o setor de saúde no Brasil são fragmentadas, resultantes da atividade compartimentada de diferentes instituições que atuam no setor ${ }^{2}$. Além disso, há poucos sistemas de informação direcionados ao gerenciamento de informações sobre a força de trabalho ou recursos humanos em saúde, sendo, em sua maioria, sistemas direcionados a análise epidemiológica, demográfica e de análise das condições de saúde. 
Este trabalho apresenta os principais resultados de uma revisão na literatura nacional e internacional, entre 2005 e 2015, sobre o tema "Sistemas de informação que apoiam à gestão de recursos humanos em saúde", tendo como seu objetivo principal identificar quais sistemas de informação foram desenvolvidos para apoiar a gestão de recursos humanos em saúde. Por fim, este artigo também apresenta a Plataforma de Recursos Humanos em Saúde, Plataforma RH, desenvolvida para subsidiar a formulação de políticas de gestão, de formação, de qualidade e de regulação da provisão de profissionais da saúde no Brasil.

Este artigo está organizado da seguinte forma: introdução, procedimentos metodológicos utilizados no estudo, resultados, apresentação da Plataforma $\mathrm{RH}$ e as considerações finais.

\section{PROCEDIMENTOS METODOLÓGICOS}

A pesquisa desenvolvida pode ser classificada como bibliográfica, uma vez que se trata de um estudo realizado por meio da análise de artigos, monografias, dissertações de mestrado e teses de doutorado sobre Sistemas de Informação que apoiam a gestão de recursos humanos em saúde, no âmbito nacional e internacional. É também uma pesquisa exploratória com abordagem quantitativa por ser um estudo que busca desenvolver e esclarecer ideias, culminando em gráficos e/ou tabelas construídos a partir de distribuições de frequência.

Segundo o esquema da pesquisa, demonstrado na Figura 1, para responder os objetivos pretendidos, a pesquisa foi dividida em duas fases: (1) Revisão bibliográfica e (2) Análise detalhada do conteúdo.
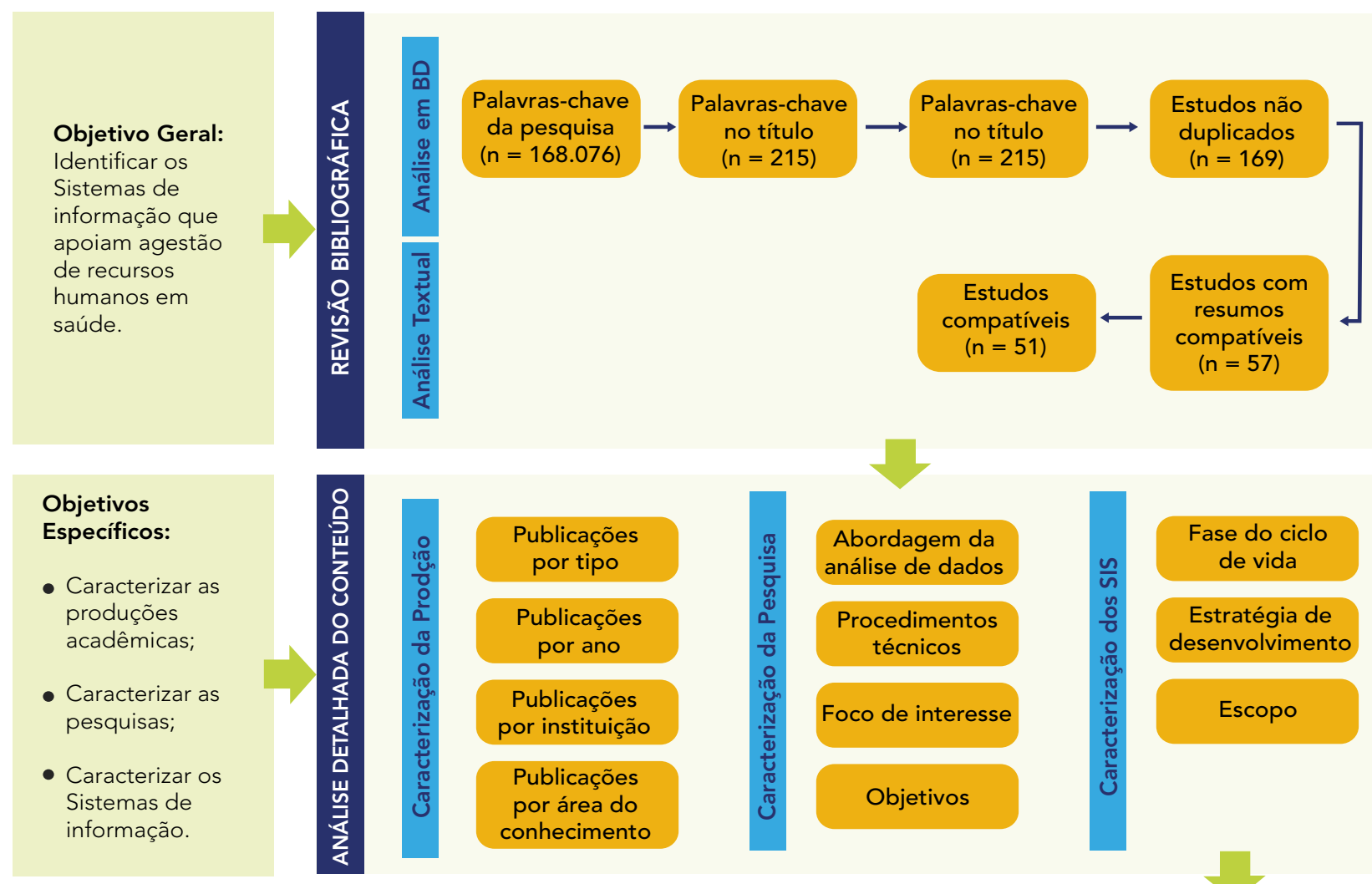
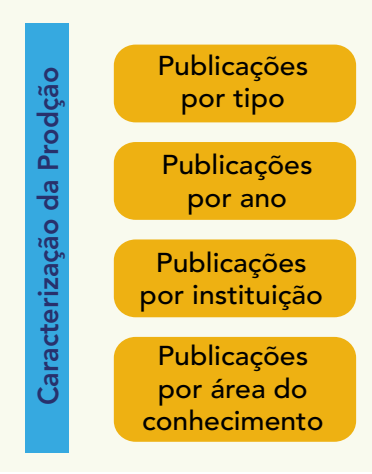

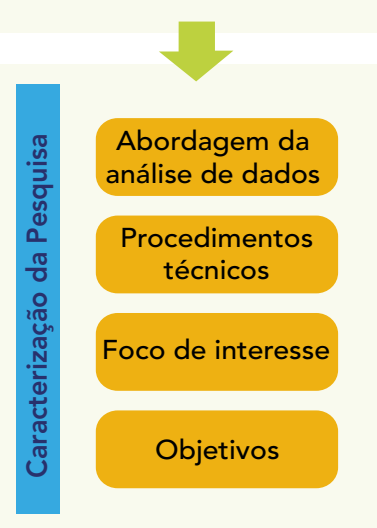

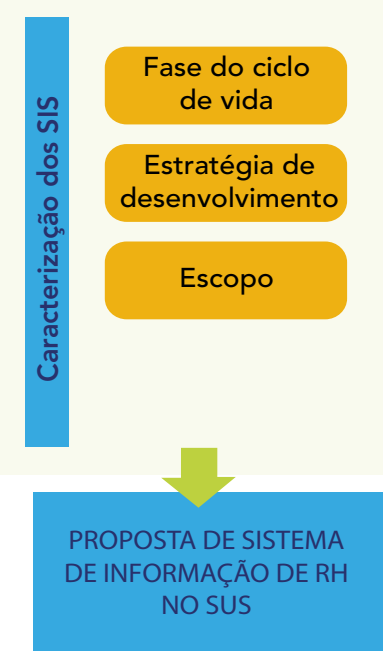

Figura 1 - Esquema da Pesquisa. 
A fase de Revisão bibliográfica foi dividida em duas subfases: Análise em banco de dados e Análise textual. Antes de descrever a fase de Análise em banco de dados é importante comentar que foram analisados artigos científicos publicados em revistas científicas ou em anais de congressos, assim como monografias, dissertações de mestrado e teses de doutorado. Para tanto, foi utilizada a ferramenta de pesquisa Google Scholar, além das bases de dados Scopus e - Portal de Periódicos da Capes/MEC.

Com o intuito de aumentar a sensibilidade da pesquisa foram utilizados vários descritores, visando selecionar trabalhos relevantes, publicados entre 2005 e 2015, tanto no idioma Português, quanto no Inglês, conforme Tabela 1:

Tabela 1 - Descritores da Pesquisa.

\begin{tabular}{l|l}
\hline Português & Inglês \\
\hline Recursos humanos e Sistema de Informação & Health and manegement and information system \\
\hline Gestão de recursos humanos e Sistema de Informação & human resources and information system \\
\hline Dados em saúde e Sistema de Informação & human resources management and information system \\
\hline Profissionais de saúde e Sistema de Informação & health data and information system \\
\hline
\end{tabular}

Conforme Figura1, a subfase de Análise em banco de dados foi realizada usando quatro critérios. O primeiro critério foi o resultado da busca conter os descritores, apresentados na Tabela 1, em qualquer parte do texto, resultando em 168.076 resultados, sendo 87.285 trabalhos oriundos de banco de dados nacionais (via Google Scholar e Portal Capes) e 80.791 de banco de dados internacionais (via Scopus). O segundo critério foi a presença dos descritores desta pesquisa exclusivamente no título dos trabalhos, resultando em 215 estudos para análise. Em seguida, a disponibilidade dos estudos foi analisada, compondo o terceiro critério, o que resultou em 194 estudos disponíveis integralmente para leitura. $\bigcirc$ quarto, e último critério, da subfase, Análise em banco de dados, foi a não duplicidade do estudo, resultando, assim, em 169 trabalhos.

Finalizada a Análise em banco de dados, iniciou-se a subfase Análise textual. Os resumos dos 169 trabalhos resultantes foram lidos e, em seguida, selecionados apenas 57 para a leitura do texto completo. Vale ressaltar que os trabalhos descartados, embora tenham sido selecionados a partir dos descritores apresentados na Tabela1, não apresentaram em seu resumo informações sobre sistemas de informação como apoiar a gestão de recursos humanos em saúde, sendo, portanto, descartados por este motivo. Após averiguação e leitura do texto completo destes 57 trabalhos, procedeu-se a exclusão de mais 6 estudos, por não apresentarem informações relevantes para a segunda fase da análise, restando, por fim, 51 textos completos para serem analisados de acordo com os critérios da fase de Análise detalhada do conteúdo.

A segunda fase da pesquisa, conforme Figura 1 foi, a análise detalhada do conteúdo, na qual os textos restantes foram lidos detaIhadamente e subdivididos de acordo com os critérios: Caracterização da produção bibliográfica, Caracterização da pesquisa científica e Caracterização do Sistema de Informação (SI). Para a caracterização da pesquisa bibliográfica, os trabalhos foram subdivididos e contabilizados por tipo de publicação, envolvendo análise de artigos de congresso, artigos de revista, monografias, dissertações de mestrado e teses de doutorado; ano de publicação, no período de 2005 a 2015; instituição do primeiro autor e área do conhecimento em que o estudo foi realizado. Na Caracterização da pesquisa científica, foi analisada a abordagem dos estudos, qualitativa ou quantitativa, e os procedimentos da pesquisa, como documental, bibliográfica, experimental ou estudo de caso. Por fim, para caracterizar os Sistemas de Informação dos trabalhos estudados, foi analisada a fase do ciclo de vida do sistema, caracterizando-o como sistema em implantação, em desenvolvimento, em produção ou como sistema legado, bem como foi caracterizado também a sua estratégia de desenvolvimento, como sendo desktop, web ou mobile. 
Por fim, a partir dos resultados encontrados, após a fase de Análise detalhada dos conteúdos, apresentar-se-á uma proposta de Sistema de Informação baseado na web para apoiar a gestão de recursos humanos em saúde, englobando funcionalidades adicionais às apresentadas pelos sistemas de informações em estudo de modo a contribuir com a literatura existente sobre o tema estudado.

\section{RESULTADOS}

Nesta seção, serão apresentados os resultados obtidos a partir da análise dos trabalhos pesquisados, a qual compreende a caracterização da produção bibliográfica, a caracterização da pesquisa científica e a caracterização dos Sistemas de Informação.

\section{CARACTERIZAÇÃO DA PRODUÇÃO BIBLIOGRÁFICA}

A produção bibliográfica dos trabalhos estudados foi caracterizada segundo o tipo de publicação, o ano de publicação, a instituição do primeiro autor e área de interesse do trabalho.

Os tipos de publicação dos artigos estudados foram resumidos na Tabela 2, assim como a quantidade encontrada de trabaIhos nacionais ou internacionais para cada tipo e seus respectivos percentuais.

Tabela 2 - Produção bibliográfica por tipo de publicação.

\begin{tabular}{l|l|l|l|l|l|l}
\hline \multirow{2}{*}{ Tipo de Publicação } & \multicolumn{2}{l}{ Quantidade } & $\%$ & \multicolumn{2}{l}{ Total } \\
\cline { 2 - 7 } & Nac. & Inter. & Total & Nac. & Inter. & (19 \\
\hline Artigos de Revista & 23 & 40 & $45,09 \%$ & $37,25 \%$ & $82,35 \%$ \\
\hline Dissertações & 3 & 0 & 3 & $5,88 \%$ & $0 \%$ & $5,88 \%$ \\
\hline Teses & 4 & 0 & 4 & $7,84 \%$ & $0 \%$ & $7,84 \%$ \\
\hline Monografias & 1 & 0 & 1 & $1,96 \%$ & $0 \%$ & $1,96 \%$ \\
\hline Artigos de Anais de Congressos & 1 & 0 & 1 & $1,96 \%$ & $0 \%$ & $1,96 \%$ \\
\hline Total & 32 & 19 & 51 & $62,74 \%$ & $37,25 \%$ & $100 \%$ \\
\hline
\end{tabular}

Segundo dados exibidos na Tabela 2, dentre os trabalhos pesquisados sobre sistemas de informação que apoiam à gestão de recursos em humanos em saúde, foram encontrados os seguintes tipos de publicação: Artigos em revista, dissertações de mestrado, teses de doutorado, monografias e artigos publicados em anais de congressos científicos. Dos 51 trabalhos estudados, 32 foram nacionais $(62,74 \%)$, enquanto 19 foram internacionais $(37,25 \%)$, sendo a grande maioria desses trabalhos representados por artigo publicado em revista (82,35\%). Foram encontradas 3 dissertações de mestrado (5,88\%), 4 teses de doutorado (7,84\%), 1 monografia $(1,96 \%)$ e 1 artigo publicado em anal de congresso $(1,96 \%)$ versando sobre o tema em estudo, no âmbito nacional. Vale destacar que, utilizando o banco de dados Scopus, foram encontrados na literatura internacional somente artigos publicados em revista, incluindo os artigos de revisão.

A pesquisa foi realizada tomando-se como critério de escolha a pesquisa dos sistemas de informação de apoio à gestão de recursos humanos em saúde nos últimos 10 anos, ou seja, foram analisadas as publicações do ano de 2005 a 2015. Os anos de publicação dos artigos analisados foram resumidos na Tabela 3, bem como a quantidade e os valores percentuais de trabalhos nacionais e internacionais encontrados. 
Tabela 3 - Produção bibliográfica por ano de publicação.

\begin{tabular}{|c|c|c|c|c|c|c|}
\hline \multirow{2}{*}{ Ano de publicação } & \multicolumn{3}{|c|}{ Quantidade } & \multicolumn{3}{|l|}{$\%$} \\
\hline & Nac. & Inter. & Total & Nac. & Inter. & Total \\
\hline 2005 & 1 & 0 & 1 & $1,96 \%$ & $0 \%$ & $1,96 \%$ \\
\hline 2007 & 1 & 1 & 2 & $1,96 \%$ & $1,96 \%$ & $3,92 \%$ \\
\hline 2008 & 1 & 0 & 1 & $1,96 \%$ & $0 \%$ & $1,96 \%$ \\
\hline 2009 & 10 & 0 & 10 & $19,6 \%$ & $0 \%$ & $19,6 \%$ \\
\hline 2010 & 6 & 3 & 9 & $11,76 \%$ & $5,88 \%$ & $17,64 \%$ \\
\hline 2011 & 3 & 3 & 6 & $5,88 \%$ & $5,88 \%$ & $11,76 \%$ \\
\hline 2012 & 2 & 1 & 3 & $3,92 \%$ & $1,96 \%$ & $5,88 \%$ \\
\hline 2013 & 4 & 8 & 12 & $7,84 \%$ & $15,68 \%$ & $23,52 \%$ \\
\hline 2014 & 4 & 1 & 5 & $7,84 \%$ & $1,96 \%$ & $9,8 \%$ \\
\hline 2015 & 0 & 2 & 2 & $0 \%$ & $3,92 \%$ & $3,92 \%$ \\
\hline Total & 32 & 19 & 51 & $62,74 \%$ & $37,25 \%$ & $100 \%$ \\
\hline
\end{tabular}

De acordo com os dados apresentados na Tabela 3, dos 10 anos pesquisados, os anos de 2009, 2010 e 2013 se destacaram como os períodos com maior número de publicações no assunto pesquisado. No ano de 2009, houve a publicação de 10 trabalhos nacionais $(19,6 \%)$, seguido pelo ano de 2010, com 6 trabalhos nacionais $(11,76 \%)$ e 3 internacionais $(5,88 \%)$, totalizando 9 trabalhos publicados (17,64\%). Em 2013, foram encontrados 4 trabalhos nacionais $(7,84 \%)$ e 8 internacionais (15,68\%), totalizando 12 estudos encontrados (23,52\%) para este ano. Nos três anos comentados, foram publicados 31 estudos $(60,76 \%)$, correspondendo a porcentagem de mais da metade do total dos trabalhos encontrados. Os anos de 2005 e 2008 apresentaram somente 1 publicação (1,96\%), enquanto os anos de 2007 e 2015 apresentaram 2 publicações (3,92\%).

$\mathrm{Na}$ Figura 2, estão representadas as publicações nacionais por instituição de origem do primeiro autor, bem como a quantidade de trabalhos encontrados por instituição.

Quantidade de Artigos

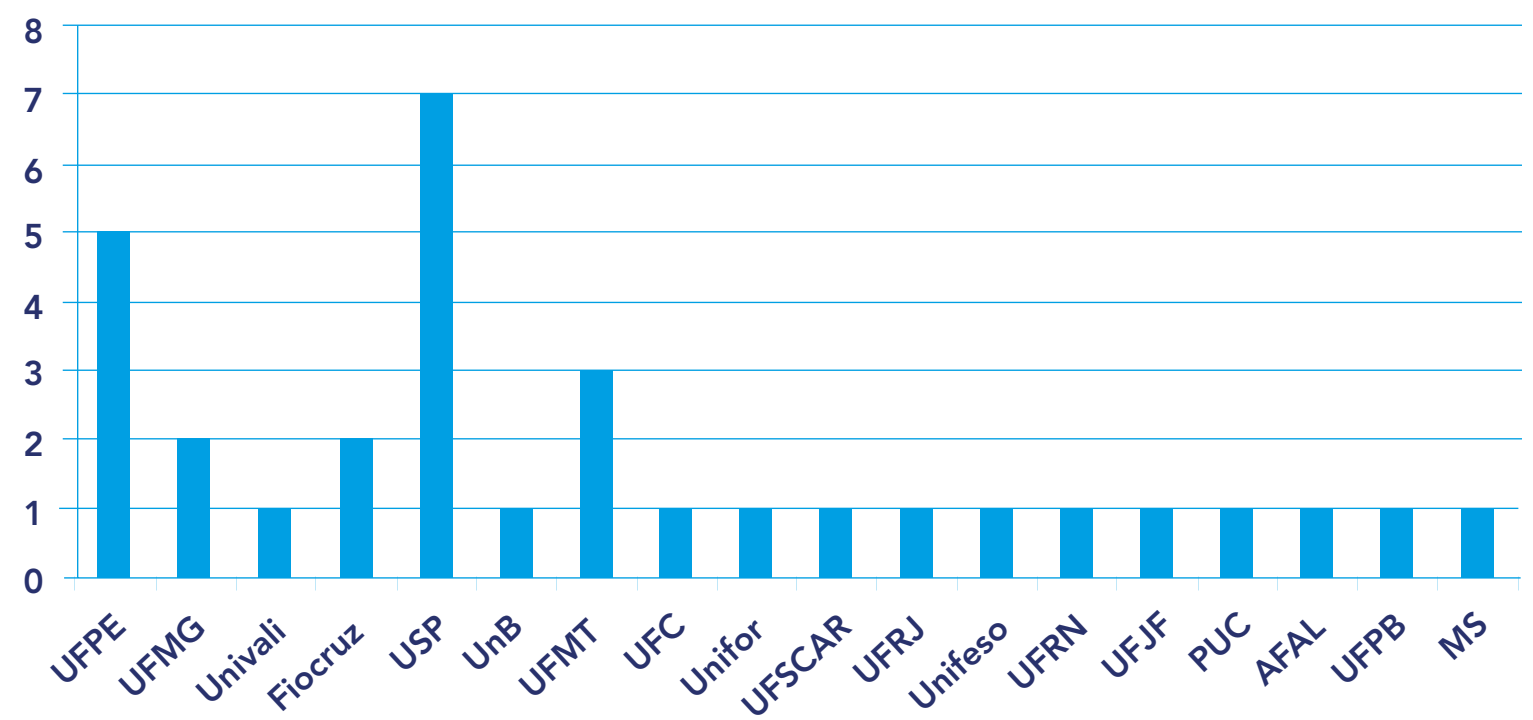

Figura 2 - Publicações bibliográficas nacionais por instituição. 
Segundo dados exibidos na Figura 2, a instituição que apresentou a maior quantidade de publicações foi a Universidade de São Paulo, com 7 trabalhos encontrados durante o processo de revisão da literatura. Desta totalidade, foram 2 artigos científicos ${ }^{12,34}, 1$ dissertação de mestrado ${ }^{39}$ e 4 teses de doutorado $33,35,36,52$. A Universidade de Pernambuco também se destacou, publicando 5 trabalhos, sendo 3 artigos científicos ${ }^{28,46,22}, 1$ monografia ${ }^{15}$ e 1 dissertação de mestrado ${ }^{46}$. A Universidade de Mato Grosso publicou 3 estudos, sendo 2 artigos científicos ${ }^{53,21}$ e 1 dissertação de mestrado ${ }^{30}$. A Universidade Federal de Minas Gerais e a Fundação Oswaldo Cruz, cada uma, publicou 2 estudos. As demais universidades, presentes na pesquisa, publicaram apenas 1 trabalho.

$\mathrm{Na}$ Tabela 4, estão representadas as publicações internacionais por instituição de origem do primeiro autor, país e a quantidade de trabalhos por instituição.

Tabela 4 - Publicações bibliográficas internacionais por instituição.

\begin{tabular}{l|l|c} 
Instituição & País & $\begin{array}{c}\text { Quantidade } \\
\text { de Artigos }\end{array}$ \\
\hline University of Washington & Estados Unidos & 2 \\
\hline IntraHealth International & Estados Unidos & 1 \\
\hline Center for Global Health & Estados Unidos & 1 \\
\hline Kennesaw State University & Estados Unidos & 1 \\
\hline Centers for Disease Control and Prevention & Estados Unidos & 1 \\
\hline Universidade Lusófona & Portugal & 1 \\
\hline Instituto Superior de Economia e Gestão & Portugal & 1 \\
\hline Universidade Lusíada & Portugal & 1 \\
\hline Government Medical College & Índia & 1 \\
\hline India Institute of Medical Sciences & Índia & 1 \\
\hline LEAD Pakistan & Paquistão & 1 \\
\hline Health Services Academy & Paquistão & 1 \\
\hline Kyung Hee University & Coréia do Sul & 1 \\
\hline The Catholic University of Korea & Coreia do Sul & 1 \\
\hline University of Bradford & Reino Unido & 1 \\
\hline Training Centre International Health & Tanzânia & 1 \\
\hline Business School & China & 1 \\
\hline
\end{tabular}

Segundo os dados apresentados na Tabela 4, entre as 19 publicações internacionais analisadas, 6 foram originadas de Universidades e Instituições dos Estados Unidos, com destaque para a Universidade de Washington, que apresentou dois artigos científicos ${ }^{38,55}$. Portugal também se destacou, apresentando 3 instituições de origem do primeiro autor, seguida da Índia, do Paquistão e da Coréia do Sul, que apresentaram 2 instituições de origem.
Reino Unido, Tanzânia, China e Finlândia apresentaram somente 1 instituição de origem de trabalho científico versando sobre o tema em estudo.

As publicações bibliográficas por área do conhecimento foram resumidas na Tabela 5, bem como a quantidade de trabalhos nacionais e internacionais encontrados e suas percentagens correspondentes. 
Tabela 5 - Publicações bibliográficas por área do conhecimento.

\begin{tabular}{l|c|l|l|c|c|c}
\hline \multirow{2}{*}{ Área do Conhecimento } & \multicolumn{2}{l|}{ Quantidade } & \multicolumn{2}{l}{ \% } \\
\cline { 2 - 7 } & Nac. & Inter. & Total & Nac. & Inter. & Total \\
\hline Ciências da Saúde & 27 & 14 & 41 & $52,94 \%$ & $27,45 \%$ & $80,39 \%$ \\
\hline Administração & 1 & 3 & 4 & $1,96 \%$ & $5,88 \%$ & $7,84 \%$ \\
\hline Engenharia de Produção & 3 & 0 & 3 & $5,88 \%$ & $0 \%$ & $5,88 \%$ \\
\hline Informática & 0 & 1 & 1 & $0 \%$ & $1,96 \%$ & $1,96 \%$ \\
\hline Engenharia da Computação & 0 & 1 & 1 & $0 \%$ & $1,96 \%$ & $1,96 \%$ \\
\hline Tecnologia da Informação & 1 & 0 & 1 & $1,96 \%$ & $0 \%$ & $1,96 \%$ \\
\hline Total & 32 & 19 & 51 & $62,74 \%$ & $37,25 \%$ & $100 \%$ \\
\hline
\end{tabular}

De acordo com os dados apresentados na Tabela 5, foram encontradas publicações referentes às seguintes áreas do conhecimento: Ciências da Saúde, Administração, Engenharia de Produção, Informática, Engenharia da Computação e Tecnologia da Informação. Quanto à distribuição da produção bibliográfica por área do conhecimento, verificou-se $80,39 \%$ dos estudos encontrados eram provenientes da área Ciências da Saúde, totalizando 41 manuscritos dos 51 trabalhos analisados, sendo 27 nacionais (52,94\%) e 14 internacionais (27,45\%). A área do conhecimento Administração apresentou 4 trabalhos $(7,84 \%)$, sendo 1 nacional ${ }^{28}$ $(1,96 \%)$ e 3 internacionais ${ }^{13,56,5}$ (5,88\%). Na área Engenharia de Produção, foram encontrados 3 trabalhos, sendo os 3 nacionais ${ }^{46,9,56}$ e, por último, Informática ${ }^{43}$, Engenharia da Computação ${ }^{29}$ e Tecnologia da Informação ${ }^{25}$ apresentaram um único estudo (1,96\%).

\section{CARACTERIZAÇÃO DA PESQUISA CIENTTÍFICA}

A segunda análise teve como objetivo caracterizar os estudos quanto: a abordagem da análise de dados, diferenciando-o em pesquisa qualitativa e em pesquisa quantitativa; aos focos de interesse; aos procedimentos técnicos, como estudo bibliográfico, estudo de caso ou documental, e aos objetivos da pesquisa, como exploratória, descritiva ou explicativa.

A caracterização da pesquisa científica de acordo com a abordagem da análise de dados está representada na Tabela 6 , bem como a quantidade e percentuais de trabaIhos nacionais e internacionais encontrados.

Tabela 6 - Pesquisa científica por abordagem da análise de dados.

\begin{tabular}{|c|c|c|c|c|c|c|}
\hline \multirow{2}{*}{$\begin{array}{l}\text { Abordagem de } \\
\text { análise }\end{array}$} & \multicolumn{3}{|c|}{ Quantidade } & \multicolumn{3}{|l|}{$\%$} \\
\hline & Nac. & Inter. & Total & Nac. & Inter. & Total \\
\hline Qualitativo & 20 & 17 & 37 & $39,21 \%$ & $33,33 \%$ & $72,54 \%$ \\
\hline Quantitativo & 12 & 2 & 14 & $23,52 \%$ & $3,92 \%$ & $27,45 \%$ \\
\hline Total & 32 & 19 & 51 & $62,74 \%$ & $37,25 \%$ & $100 \%$ \\
\hline
\end{tabular}

Segundo dados exibidos na Tabela 6, dos 51 trabalhos encontrados, 37 utilizaram abordagem qualitativa (72,54\%), sendo 20 nacionais $(39,21 \%)$ e 17 internacionais (33,33\%), e empregando a abordagem quantitativa, foram encontrados 14 estudos (27,45\%), sendo 12 nacionais $(23,52 \%)$ e 2 internacionais $(3,92 \%)$. Logo, conclui-se que grande parte dos estudos sobre o tema, Sistemas de informação como apoio à gestão de recursos humanos em saúde, utilizam análise qualitativa, ou seja, não faz uso de análise estatística. 
A caracterização dos estudos por foco de interesse está representada na Figura 3. Para delimitação desses focos, foi realizada a leitura completa dos textos e, em seguida, foram avaliados seus objetivos gerais. Por fim, os trabalhos foram categorizados em sete focos de interesses principais: avaliação de sistema de informação, importância de sistema de informação, análise de dados produzidos por sistema de informação, revisão da literatura, aperfeiçoamento de sistema de informação, implantação de sistema de informação e desenvolvimento de sistema de informação.

\section{Quantidade de Trabalhos}

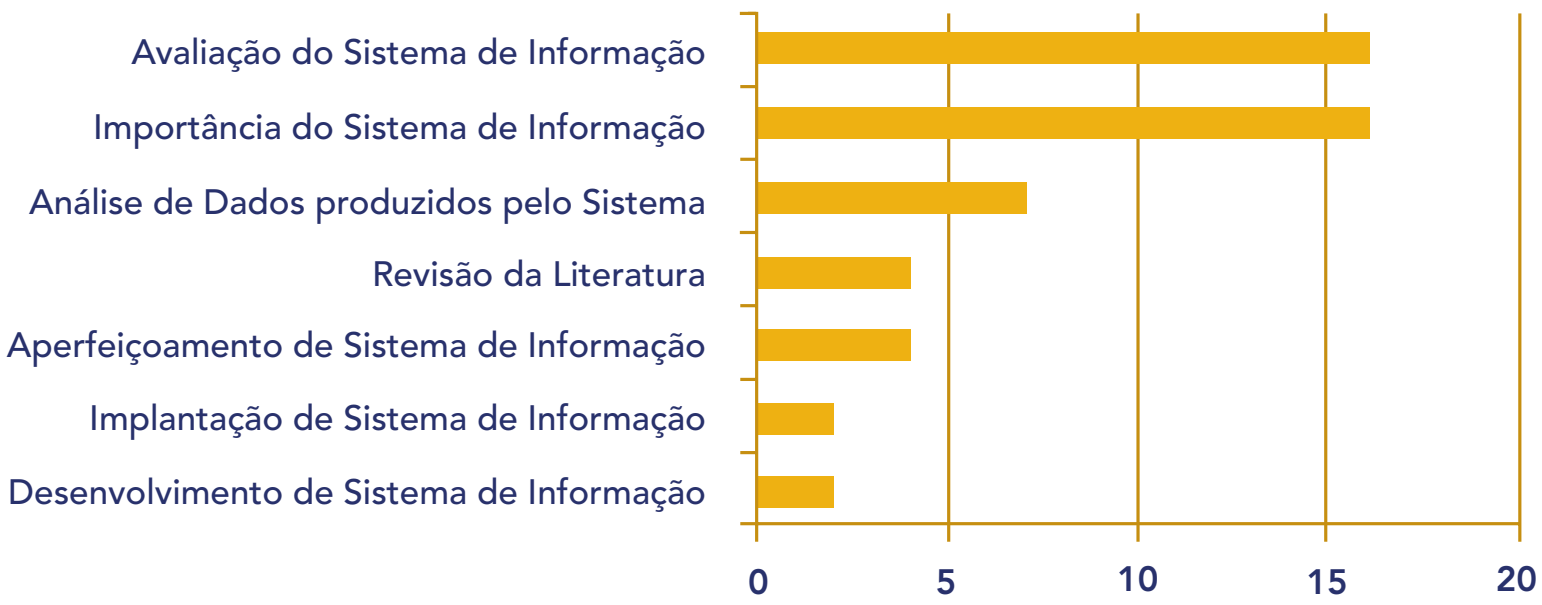

Figura 3 - Pesquisa científica por foco de interesse.

De acordo com os dados exibidos na Figura 3, a maioria dos trabalhos apresentou como foco de interesse o estudo da "Avaliação de um sistema de informação" e o estudo da "Importância de um sistema de informação" em determinado contexto, contabilizando 16 trabalhos em cada um destes focos no universo de 51 trabalhos pesquisados. No tocante ao foco "Avaliação de sistema de informação", os trabalhos abordaram as seguintes questões: avaliação do processo de trabalho e geração de dados, avaliação da qualidade do preenchimento dos dados e da informação inserida no sistema e, ainda, avaliação dos processos de produção de dados e informações. Por outro lado, com respeito ao foco "Importância de um sistema de informação", os trabalhos versaram, em sua maioria, sobre a contribuição dos sistemas para a disseminação de informação ou sobre a implementação de melhorias na gestão em saúde.

○ foco de interesse "Análise de dados produzidos por um sistema de informação" esteve presente em 7 trabalhos pesquisados. Tal análise envolveu dados ambulatoriais, hospitalares, de atenção básica, agravos de notificação, de mortalidade, de vigilância alimentar, nutricional, financeira e de recursos humanos. $O$ aperfeiçoamento de bases de dados do sistema de informação ou melhoramento de funcionalidades do sistema foi o foco de interesse de 4 trabalhos. Igualmente, 4 artigos apresentaram como foco de interesse a "Revisão da literatura" sobre sistemas de informações. Por último, os focos "Implantação de sistema de informação" e "Desenvolvimento de sistema de informação" foram almejados em somente dois artigos, cada um.

A caracterização da pesquisa científica quanto aos procedimentos técnicos estão apresentados na Tabela 7, assim como a quantidade encontrada de trabalhos nacionais ou internacionais e seus respectivos percentuais. 
Tabela 7 - Pesquisa científica quanto aos procedimentos técnicos.

\begin{tabular}{|c|c|c|c|c|c|c|}
\hline \multirow{2}{*}{$\begin{array}{l}\text { Procedimentos } \\
\text { técnicos }\end{array}$} & \multicolumn{3}{|c|}{ Quantidade } & \multicolumn{3}{|l|}{$\%$} \\
\hline & \multirow{2}{*}{$\begin{array}{r}\text { Nac. } \\
25\end{array}$} & \multirow{2}{*}{\begin{tabular}{|r} 
Inter. \\
16 \\
\end{tabular}} & \multirow{2}{*}{$\begin{array}{r}\text { Total } \\
41 \\
\end{array}$} & \multirow{2}{*}{\begin{tabular}{|l|} 
Nac. \\
$49,01 \%$ \\
\end{tabular}} & \multirow{2}{*}{$\begin{array}{l}\text { Inter. } \\
31,37 \% \\
\end{array}$} & \multirow{2}{*}{$\begin{array}{l}\text { Total } \\
80,39 \%\end{array}$} \\
\hline Bibliográfico & & & & & & \\
\hline Estudo de Caso & 4 & 3 & 7 & $7,84 \%$ & $5,88 \%$ & $13,72 \%$ \\
\hline Documental & 3 & 0 & 3 & $5,88 \%$ & $0 \%$ & $5,88 \%$ \\
\hline Total & 32 & 19 & 51 & $62,74 \%$ & $37,25 \%$ & $100 \%$ \\
\hline
\end{tabular}

Para a realização da caracterização da pesquisa científica, demonstrada na Tabela 7, os estudos foram analisados e subdivididos de acordo com seus procedimentos técnicos: estudo bibliográfico, estudo de caso ou documental. Os trabalhos utilizaram predominantemente a pesquisa bibliográfica como procedimento técnico, compreenderam 41 estudos (80,39\%), compostos por 25 trabalhos nacionais $(49,01 \%)$ e 16 internacionais $(31,37 \%)$.

No tocante ao procedimento técnico "Estudo de Caso", foram encontrados 7 estudos utilizando este procedimento $(13,72 \%)$, sendo 4 nacionais $(7,84 \%)$ e 3 internacionais (5,88\%). Analisando o conteúdo detalhado destes estudos de casos, percebeu-se que seus objetivos gerais tratavam três temas principais: a análise do ambiente hospitalar após a implantação do sistema de informação ${ }^{5}$, a análise do processo de produção de dados e informações pelo sistema de informação ${ }^{30}$ e o estudo de características dos processos de gestão auxiliados pelo uso de sistemas de informações 53,43,29,22. $^{\text {. }}$

Para finalizar a explicação dos dados da Tabela 7, têm-se os estudos que utilizaram como procedimento técnico a pesquisa do tipo documental. Nos 51 trabalhos analisados, 3 estudos nacionais utilizaram documentos como base para a sua realização $(5,88 \%)$. Os documentos utilizados nas pesquisas nacionais foram: documento de implantação do Sistema de Informação de Gestão de Recursos Humanos em Saúde SIGRHS ${ }^{15}$, norma ISSO $27001^{39}$, documentos do Sistema de Informação da Atenção Básica - SIAB, Sistema de Informação sobre Mortalidade (SIM) e Sistema de Informação do Programa Nacional de Imunização (SI-PNI) ${ }^{36}$.

A caracterização da pesquisa científica quanto aos objetivos está demonstrada na Tabela 8. Os resultados dos objetivos da pesquisa foram categorizados em: objetivos exploratórios, descritivos, explicativos e indefinidos. O termo "indefinido" foi incluído como categoria para representar os trabalhos que não identificaram os seus objetivos da pesquisa como estudo exploratório, descritivo ou explicativo.

Tabela 8 - Pesquisa científica quanto aos objetivos da pesquisa.

\begin{tabular}{l|c|c|c|c|c|c}
\hline \multirow{2}{*}{$\begin{array}{l}\text { Objetivos da } \\
\text { pesquisa }\end{array}$} & \multicolumn{2}{l|}{ Quantidade } & \multicolumn{2}{l|}{$\%$} \\
\cline { 2 - 7 } & Nac. & \multicolumn{1}{l|}{ Inter. } & \multicolumn{1}{l|}{ Total } & Nac. & Inter. & Total \\
\cline { 1 - 7 } Exploratório & 3 & 0 & 3 & $5,88 \%$ & $0 \%$ & $5,88 \%$ \\
\hline Descritivo & 7 & 0 & 7 & $13,72 \%$ & $0 \%$ & $13,72 \%$ \\
\hline Explicativo & 0 & 0 & 0 & $0 \%$ & $0 \%$ & $0 \%$ \\
\hline Indefinido & 22 & 19 & 41 & $43,13 \%$ & $37,35 \%$ & $80,39 \%$ \\
\hline Total & 32 & 19 & 51 & $62,74 \%$ & $37,25 \%$ & $100 \%$ \\
\hline
\end{tabular}

Conforme a Tabela 8, dentre os 51 trabaIhos analisados, 41 não identificaram o objetivo da pesquisa (80,39\%), enquanto somente 10 estudos classificaram suas pesquisas como exploratórias (5,88\%) ou descritivas (13,72\%). Porém, nenhum trabalho internacional analisado definiu tal objetivo, podendo esboçar, assim, que as pesquisas na área de sistemas 
de informação como apoio à gestão de recursos humanos em saúde não apresentaram rigor metodológico, buscando definir claramente a metodologia da pesquisa utilizada ou, então, que se está buscando familiaridade com o tema, visto que pode ser considerado pouco explorado.

\section{CARACTERIZACÃO DOS SISTEMAS DE INFORMAÇẪO}

procedimento de caracterização dos Sistemas de Informações foi realizado através de três análises principais: avaliação do escopo do sistema de informação, verificação da fase do ciclo de vida do sistema de informação e análise da estratégia de desenvolvimento do sistema de informação. Por se tratar, neste tópico, da seção caracterização dos sistemas de informação, serão considerados somente os 22 trabalhos, dentro do universo desta pesquisa, que estudam um sistema específico.

$\mathrm{Na}$ Tabela 9, estão representados todos os sistemas de informações presentes nos 22 trabalhos analisados, incluindo sistemas nacionais e internacionais. Cada linha da tabela é composta pelo nome do sistema, pelo seu escopo e pelo número de ocorrência desse sistema nos estudos analisados.

Tabela 9 - Sistemas de informação por escopo.

\begin{tabular}{l|l|c}
\hline Sistema de Informação & Escopo & Ocorrência \\
\hline Sistema de Informações Ambulatoriais & Ambulatorial & 1 \\
\hline $\begin{array}{l}\text { Rede de Observatórios de Recursos Humanos } \\
\text { das Américas }\end{array}$ & Produção acadêmica em RH & 1 \\
\hline Sistema de Informações Hospitalares & Hospitalar & 3 \\
\hline Sistema de Informação da Atenção Básica & Atenção básica & 6 \\
\hline Sistema de Informação sobre Mortalidade & Mortalidade & 6 \\
\hline Sistema de Informação sobre Nascidos Vivos & Nascidos vivos & 3 \\
\hline Sistema de Acompanhamento da Gestante & Gestante & 1 \\
\hline $\begin{array}{l}\text { Sistema de Informação sobre Agravos de Notifi- } \\
\text { cação }\end{array}$ & Agravos de notificação & 2 \\
\hline $\begin{array}{l}\text { Sistema de Cadastramento e Acompanhamento } \\
\text { de Hipertensos e Diabéticos }\end{array}$ & Hipertensão e diabetes \\
\hline $\begin{array}{l}\text { Sistema de Informação e Gestão de Recursos } \\
\text { Humanos em Saúde }\end{array}$ & Gestão de RH & 1 \\
\hline $\begin{array}{l}\text { Sistema de Informação para Assistência Oncoló- } \\
\text { gica }\end{array}$ & Dados oncológicos & 1 \\
\hline Sistema de Gestão de Segurança da Informação & Segurança da Informação & 1 \\
\hline Registro de Câncer de Base Populacional & Registros de Câncer & 1 \\
\hline Sistema de Vigilância Alimentar e Nutricional & Dados alimentares e nutricionais & 1 \\
\hline Hygia Web - Gestão da Saúde Pública & Gestão da saúde pública & 1 \\
\hline $\begin{array}{l}\text { Sistema Informatizado do Hospital das Clínicas da } \\
\text { FMRB }\end{array}$ & Hospitalar & 1 \\
\hline $\begin{array}{l}\text { Sistema de Monitoramento e Avaliação do Pré- } \\
\text {-natal, parto, puerpério e Criança }\end{array}$ & $\begin{array}{l}\text { Pré-natal, parto, puerpério e de } \\
\text { crianças }\end{array}$ & 1 \\
\hline $\begin{array}{l}\text { Sistema Finanças do Brasil } \\
\text { Sistema de Informação de Orçamentos Públicos }\end{array}$ & Financeiro & 1 \\
\hline
\end{tabular}


Como pode ser observado, mediante a revisão da literatura, o Sistema de Informação de Atenção Básica e o Sistema de informação sobre Mortalidade foram os mais estudados nos trabalhos analisados por terem apresentado o maior número de ocorrências.

Ademais, é importante ressaltar que somente dois trabalhos citaram Sistemas de Informações desenvolvidos para apoiar a gestão de recursos humanos em saúde: a Rede Observatório de Recursos Humanos das Américas (Rede ObservaRH) ${ }^{28}$ e $\mathrm{O}$ Sistema de Informação e Gestão de Recursos Humanos em Saúde (SIGRHS) ${ }^{15}$. A Rede ObservaRH reúne a produção acadêmica na área de recursos humanos em saúde nas américas e o SIGRHS gerencia dados de um único hospital situado no município de Porto Seguro/BA. Os demais sistemas, retratados na Tabela 9, objetivam gerir dados pontuais da saúde, como dados ambulatoriais, de mortalidade, de câncer, dentre outros. São os sistemas considerados de apoio operacional. Em adição, também foram encontrados sistemas desenvolvidos como apoio à administração para gerenciar dados financeiros e orçamentários.

Após a verificação dos sistemas de informações presentes nos estudos, foi analisada em qual fase do ciclo de vida estavam os sistemas pesquisados com o intuito de identificar se o sistema estudado estava sendo desenvolvido, estava em fase de implantação ou em pleno funcionamento, tal como mostrado na Tabela 10.

Tabela 10 - Sistemas de Informação por fase do ciclo de vida.

\begin{tabular}{lcc}
\hline Fase do ciclo de vida & Quantidade & $\%$ \\
\hline Desenvolvimento & 1 & $4,54 \%$ \\
Implantação & 1 & $4,54 \%$ \\
Produção & 20 & $90,90 \%$ \\
Total & 22 & $100 \%$ \\
\hline
\end{tabular}

Como observado na Tabela 10, dos 22 trabalhos nacionais e internacionais analisados, 20 estão na fase de produção $(90,90 \%)$, enquanto apenas 1 estudo tratava do desenvolvimento de um sistema de informação $(4,54 \%)^{50}$ e 1 relatava sobre a implantação de um sistema de informação $(4,54 \%)^{15}$. Logo, a maioria esmagadora dos estudos analisa sistemas em funcionamento, o que pode tornar os resultados mais significativos, uma vez que sistemas em desenvolvimento ou em fase de implantação estão mais propensos a mudanças.

A caracterização do sistema de informação por estratégia de desenvolvimento permite verificar se os sistemas estudados foram desenvolvidos para funcionar via desktop, web ou mobile. O número de trabalhos e as suas estratégias de desenvolvimento estão representados na Tabela 11.

Tabela 11 - Sistemas de informação por estratégia de desenvolvimento.

\begin{tabular}{l|c|l}
\hline $\begin{array}{l}\text { Estratégia de } \\
\text { desenvolvimento }\end{array}$ & Quantidade & $\%$ \\
\hline Web & 19 & $86,36 \%$ \\
\hline Desktop & 0 & $0 \%$ \\
\hline Mobile & 0 & $0 \%$ \\
\hline Indefinido & 3 & $5,88 \%$ \\
\hline Total & 22 & $100 \%$ \\
\hline
\end{tabular}

Dentre os 22 trabalhos analisados, 19 foram desenvolvidos para funcionar via Web (86,36\%) e nenhum trabalho mencionou ser desenvolvido para desktop ou mobile. Porém, 3 destes estudos não deixaram claro qual estratégia foi utilizada. Tal constatação pode significar que os sistemas desenvolvidos no âmbito da saúde são relativamente recentes, uma vez que a grande maioria utiliza a estratégia web. Por outro lado, a não apresentação de nenhum sistema mobile sinaliza que os sistemas na área em estudo, gestão de recursos humanos em saúde, ainda não avançaram a ponto de tender às exigências modernas acerca da utilização de dispositivos móveis.

\section{PROPOSTA DE PLATAFORMA WEB DO SISTEMA ÚNICO DE SAÚDE}

Antes de descrever a Plataforma de Recursos Humanos em Saúde, Plataforma $\mathrm{RH}$, é importante mencionar que se trata de uma demanda do Ministério da Saúde, desenvolvida em parceria com o Laboratório 
de Inovação Tecnológica em Saúde da Universidade Federal do Rio Grande do Norte, LAIS/UFRN, tendo como seu principal objetivo a reunião de dados relacionados aos profissionais da saúde, no âmbito nacional, em um único repositório de dados de modo a assistir à tomada de decisão com informações corretas, completas e oportunas.

Com base nos resultados da análise dos estudos pesquisados, foram encontrados somente dois sistemas de informação que apoiam à gestão de recursos humanos em saúde no Brasil: (a) Sistema de Informação e Gestão de Recursos Humanos em Saúde, desenvolvido e implementado no hospital de Porto Seguro/BA - SIGRHS (JULIÃO; BATISTELLA, 2009) e (b) Rede Observatório de Recursos Humanos das Américas - Rede ObservaRH (MEDEIROS, 2005), instituída em 1999 por meio de parceria entre o Ministério da Saúde (MS), a Organização Pan-americana de Saúde (OPAS-OMS) e instituições nacionais de pesquisa. Porém, essas duas iniciativas de desenvolvimento de sistema contemplam apenas parcialmente as demandas atuais do Ministério da Saúde, o que culminou na construção da plataforma $\mathrm{RH}$, uma vez que o SIGRHS gerencia dados de um único hospital do país e a Rede ObservaRH enfoca somente a produção acadêmica de recursos humanos em saúde. Logo, estes dados apontam uma lacuna no desenvolvimento de sistemas de informação que apoiam à gestão de recursos humanos em saúde, especialmente no Brasil, confirmado a necessidade do Ministério da Saúde quanto ao desenvolvimento da Plataforma $\mathrm{RH}$.

A Plataforma RH é um sistema de informação baseado na web para a integração de dados da saúde nacional, criada para subsidiar a formulação de políticas de gestão, de formação, de qualidade e de regulação da provisão de profissionais da saúde no Brasil. Além disso, ela propicia o intercâmbio de informações entre pesquisadores, profissionais e alunos da área da saúde, bem como auxilia a negociação entre segmentos do governo e trabalhadores da saúde. Ela utiliza técnicas de mineração de dados com o objetivo de descobrir regras e identificar tendências, encontrando, dessa forma, relacionamentos ocultos entre os dados de modo a auxiliar a tomada de decisão estratégica. $\bigcirc$ Ministério da Saúde e seus principais departamentos e secretarias participam da construção da referida plataforma, assim como o LAIS/UFRN. Na Figura 4, está demonstrada a visão geral da Plataforma $\mathrm{RH}$.

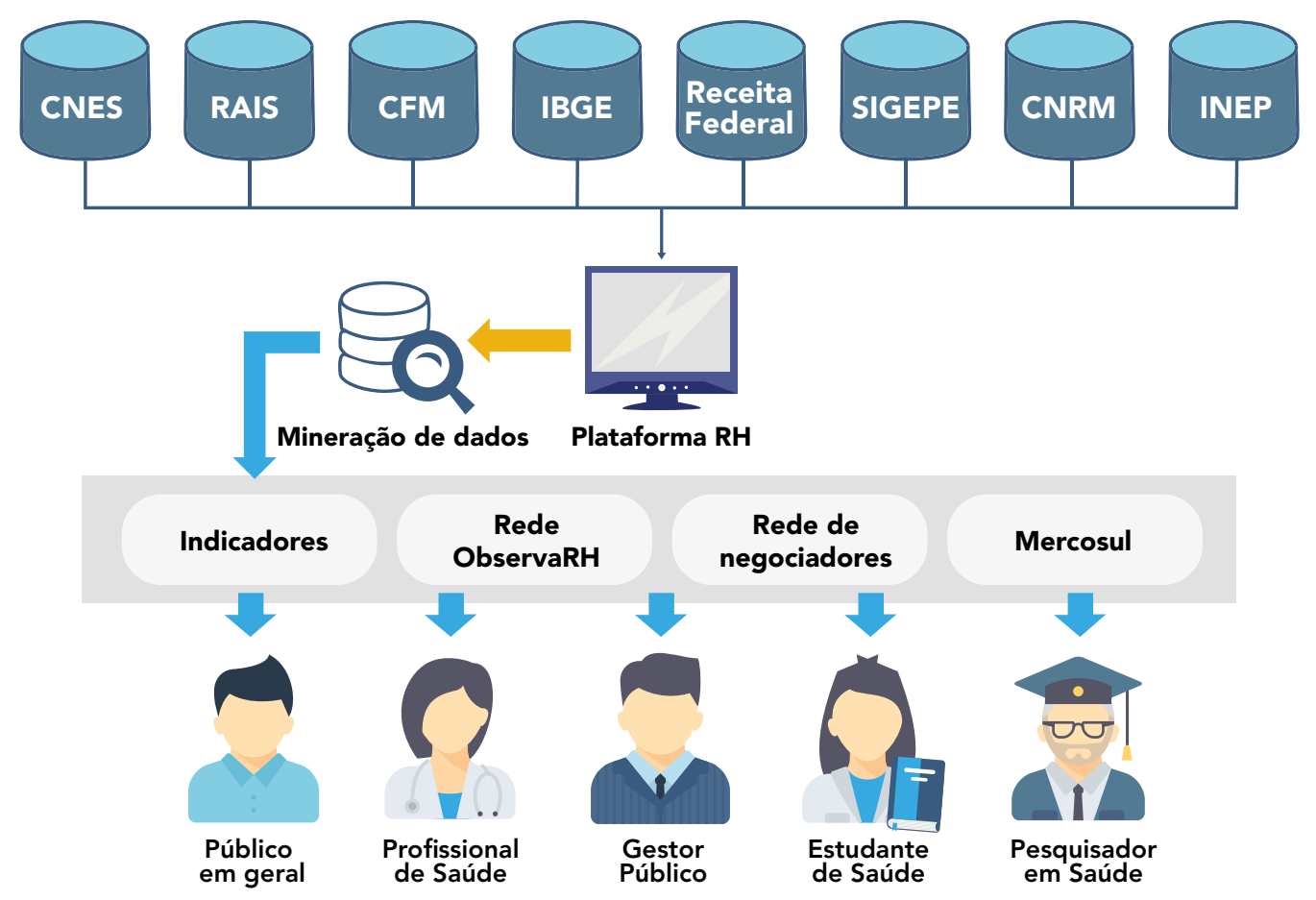

Figura 4 - Visão geral da Plataforma RH. 
De acordo com a Figura 4, as informações para a alimentação dos módulos da plataforma são obtidas a partir das seguintes instituições: (a) Cadastro Nacional de Estabelecimentos de Saúde - CNES; (b) Relação Anual de Informações - RAIS; (c) Conselho Federal de Medicina - CFM; (d) Instituto Brasileiro de Geografia $e$ Estatística - IBGE; (e) Receita Federal; (f) Sistema de Gestão de Pessoas - SIGEPE; (g) Sistema de Comissão Nacional de Médicos Residentes - CNRM e (h) Instituto Nacional de Estudos e Pesquisas Educacionais Anísio Teixeira - INEP.

Os dados destas instituições serão coletados, organizados e minerados para, posteriormente, alimentarem os módulos da Plataforma RH. São eles: Indicadores, Rede ObservaRH, Rede de Negociadores e Mercosul.

O módulo Indicadores objetiva promover a seleção, o tratamento e a oferta de dados que retratem a distribuição, a formação, a relação profissional/população, o perfil dos profissionais, as formas de inserção, o vínculo, o regime e a jornada de trabalho dos profissionais da saúde, além das características das unidades de saúde em que tais trabalhadores desempenham suas atividades. Concentra também informações sobre a formação e especialização dos profissionais da saúde, mostrando carências e convergências destes profissionais em todo o Brasil.

O módulo Rede ObservaRH é composto por 24 estações de pesquisa, sendo cada uma representada por um estado brasileiro. Ela tem como principal objetivo disponibilizar o acesso a estudos e pesquisas cientificas sobre recursos humanos em saúde, bem como propiciar o mais amplo acesso a informações e análises sobre esta área no país, facilitando a formulação, desse modo, o acompanhamento e a avaliação de políticas e programas setoriais no campo da saúde. É importante ressaltar que o módulo Rede ObservaRH deriva da Rede Observatório de Recursos Humanos em Saúde, instituída no Brasil em 1999 e comentada no início desta seção.

No módulo Mercosul, estão disponibilizas informações sobre profissionais que exercem sua profissão no MERCOSUL e/ ou trabalham em municípios ou jurisdições de fronteira. Este módulo tem como objetivo disponibilizar informações profissionais de formação, contabilização de currículos, registro profissional, regulação do trabalho e requisitos para o exercício profissional no MERCOSUL.

$\mathrm{O}$ último módulo da Plataforma $\mathrm{RH}$, módulo Rede de Negociadores, funciona como uma mesa de negociação, é a Mesa Nacional de Negociação Permanente do Sistema Único de Saúde (MNNP-SUS), um fórum paritário de negociação, que reúne gestores públicos, prestadores de serviços privados da saúde e entidades sindicais nacionais representativas dos trabalhadores da saúde. Este módulo tem como objetivo promover a troca de experiências e a construção de negociações com a finalidade de implantar novas metodologias para o aprimoramento do processo de trabalho no âmbito do SUS, bem como orientar o desenvolvimento de estratégias, visando o atendimento das demandas conforme as finalidades, os princípios e as diretrizes do Sistema Único de Saúde.

Como a proposta da Plataforma RH consiste em um sistema de informação com arquitetura Web e padrões de dados acessíveis, diferentes usuários poderão fazer uso de suas informações. Por exemplo, o público em geral terá acesso aos documentos ou publicações científicas no repositório do módulo Rede ObservaRH, também poderão acessar dados públicos dos profissionais de saúde e dos postos de trabalho do seu município ou Estado, disponíveis no módulo Indicadores; Profissionais de saúde, por sua vez, poderão participar das ferramentas de discussão do módulo Rede de Negociadores ou terem acesso aos dados de equipamentos, profissionais e de postos de trabalho, também através do módulo Indicadores; Gestores federais, estaduais ou municipais poderão utilizar as ferramentas da Plataforma $\mathrm{RH}$ para mediar fóruns de discussões na Rede de Negociadores ou, ainda, para visualizar a disponibilidade de profissionais no seu estado ou município; Estudantes em saúde poderão acessar o repositório do módulo Rede ObservaRH para analisar as publicações em saúde por estados, enquanto os Pesquisadores em saúde poderão alimentar 
a Rede ObservaRH com seus trabalhos publicados ou acessarem às publicações da Rede em outros estados.

\section{CONSIDERAÇÕES FINAIS}

Este artigo apresentou os principais resultados de uma revisão na literatura nacional e internacional, entre 2005 e 2015, efetuada por meio de artigos publicados em revista e em anais de congresso, bem como por meio de dissertações de mestrado e de teses de doutorado sobre o tema "Sistemas de informação que apoiam à gestão de recursos humanos em saúde", tendo como objetivo principal identificar quais sistemas de informação foram desenvolvidos para apoiar a gestão de recursos humanos em saúde entre os anos de 2005 e 2015. Para tanto, partiu-se da Revisão bibliográfica, composta pela análise em banco de dados e pela análise textual até a Análise detalhada do conteúdo e, por fim, foi apresentada uma proposta de sistema de informação, em desenvolvimento, para atender as demandas do Ministério da Saúde, denominada Plataforma RH do SUS.

A fase de Revisão bibliográfica resultou em 51 artigos para leitura e análise integral, dos quais foram identificados 22 sistemas de informação provendo, em algum nível, gestão de recursos humanos em saúde.

A fase de Análise detalhada do conteúdo foi composta pela caracterização da produção bibliográfica, pela caracterização da pesquisa científica e pela caracterização dos sistemas de informação.

Os principais resultados da caracterização da produção bibliográfica foram: 82,35\% dos trabalhos eram artigos científicos; a maioria dos trabalhos foram publicados em 2009, 2010 e 2013; no âmbito nacional, as instituições que mais publicaram trabalhos sobre o tema em estudo foram USP e UFPE, enquanto as universidades dos Estados Unidos, sobretudo a Universidade de Washington, foram as que mais se destacaram internacionalmente; $80,39 \%$ dos trabalhos pesquisados eram da área de Ciências da Saúde.
No processo de caracterização da pesquisa científica, foi observado que $74,02 \%$ dos trabalhos apresentaram a abordagem qualitativa para a análise de dados, enquanto $27,45 \%$ apresentaram abordagem quantitativa. Os principais focos de interesse identificados nos estudos foram "Avaliação do Sistema de Informação" e "Importância do Sistema de Informação". Quanto à avaliação dos procedimentos técnicos, 80,39\% dos trabalhos foram do tipo bibliográfico e 41 dos 51 trabalhos, não identificaram, em seu manuscrito, os objetivos da pesquisa, se exploratório, descritivo ou explicativo, ao passo que 10 trabalhos dos 51 pesquisados definiram explicitamente seu objetivo de estudo como sendo exploratório.

A caracterização dos sistemas de informação alcançou os seguintes resultados para os 22 sistemas de informação identificados nos 51 artigos analisados: 90,90\% dos sistemas foram definidos como estando em produção, ou seja, em funcionamento; $86,36 \%$ foram desenvolvidos para a plataforma web; somente 2 dos 22 sistemas de informação encontrados discutiram sobre o apoio à gestão de recursos humanos em saúde, demonstrando, assim, escassez de sistemas desenvolvidos com este fim, enquanto, por outro lado, os demais sistemas cuidavam de informações epidemiológicas, financeiras ou de gestão hospitalar.

Apesar de haver, especialmente no Brasil, diferentes Sistemas de Informação que auxiliem a gestão em saúde, ainda existem deficiências quanto ao gerenciamento de informações sobre a força de trabalho ou recursos humanos em saúde no país. Desse modo, finalizando o estudo, foi proposta e apresentada a Plataforma de Recursos Humanos em Saúde do Sistema Único de Saúde - SUS, Plataforma RH, em fase de desenvolvimento pelo Laboratório de Inovação Tecnológica em Saúde da Universidade Federal do Rio Grande do Norte, LAIS/UFRN.

A Plataforma $\mathrm{RH}$ foi apresentada como um sistema web de integração de dados da saúde nacional, criada para subsidiar a formulação de políticas de gestão, de formação, de qualificação e de regulação da 
provisão de profissionais da saúde no Brasil, o que possibilitará o preenchimento das principais lacunas relacionadas à gestão de informações sobre recursos humanos em saúde no país.

Como a Plataforma supramencionada encontra-se em fase de desenvolvimento, sugere-se como pesquisa futura o detalhamento desta plataforma, a nível conceitual e de implementação, de modo a auxiliar os pesquisadores em saúde a compreender o seu funcionamento, bem como inspirar pesquisas futuras nesta área. Em adição, pode também ser realizada uma pesquisa, em âmbito nacional, a fim de aferir a satisfação dos usuários da plataforma, o que poderá contribuir para o seu aprimoramento. 


\section{REFERÊNCIAS}

BRASIL. Ministério da Saúde. Sistema de Planejamento do SUS (PlanejaSUS): uma construção coletiva - trajetória e orientações de operacionalização. Brasília, 2009 (a).

BRASIL. Ministério da Saúde. A experiência brasileira em sistemas de informação em saúde. Fundação Oswaldo Cruz, Brasília, 2009 (b).

BENITO, G. A. V., LICHESKI, A. P. Sistema de informação apoiando a gestão do trabalho em saúde. Revista Brasileira de Enfermagem. v.62, n.3, p.447-50, 2009.

BITTENCOURT, S. A et al. Assistência a crianças desnutridas: análise de dados do Sistema de Informação Hospitalar do Sistema Único de Saúde do Brasil. Rev. Bras. Saúde Materna e Infantil. v.9, n.3, p.263-273, 2009.

CARDOSO, I. R; PEDRON, C. D. Users' commitment in information system implementation: the role of top-management commitment and organizational context. Revista de Gestão em Sistemas de Saúde. São Paulo. v. 2, n.1. p.03-25, 2013.

COELI, C. M. Sistemas de informações em saúde e uso de dados secundários na pesquisa e avaliação em saúde. Caderno de Saúde Coletiva. v. 18, n. 3, p.335-6, 2010.

$\mathrm{CHOI}, \mathrm{W}$ et al. Information system success model for customer relationship management system in health promotion centers. Healthcare Informatics Research. v.19, n2, 2013.

CORREIRA, L. O. S; PADILHA, B. M; VASCONCELOS, S. M. L. Métodos para avaliar a completitude dos dados dos sistemas de informação em saúde do Brasil: uma revisão sistemática. Ciências \& Saúde Coletiva. v. 19, n. 11, p. 4467-4478, 2014.

CHU, M. G. P. K. Proposta de Modelo de gestão Estratégica com Ênfase em Recursos Humanos e Sistemas de Informação. In: XXX ENCONTRO NACIONAL DE ENGENHARIA DE PRODUÇÃO, São Carlos, São Paulo, 2010.

DANIEL, V. M. The information system on mortality and its support to the management and planning of the Brazilian public health system (SUS). Revista de Gestão em Sistemas de Saúde. São Paulo, v. 2, n. 2, p.148-173, 2013.

DATASUS. Portal da Saúde do Sistema Único de Saúde. Disponível em: <http://www2.datasus.gov.br/DATASUS/index.php> Acesso em: 9 out. 2015.

GRACIANO, M. M. C; ARAUJO, E. W; NOGUEIRA, D. A. Sistema de Informação em saúde e atuação do profissional médico. Rev. Med. Minas Gerais. v.19, n.3, p.198-205, 2009. 
HUSSAIN, Z; WALLACE, J; CORNELIUS, N. The use and impact of human resource information systems on human resource management professionals. Information \& management. v. 44, p. 74-89, 2007.

JESUS, M. C. P et al. Atualização dos dados nos sistemas de informação em saúde. Revista de enfermagem. v.20, n.2, p.795-801, 2012.

JULIÃO, M. C. A; BATISTELLA, D. C. S. Processo de implantação do Sistema de Informação e Gestão de Recursos Humanos em Saúde - SIGRHS no Hospital Municipal de Porto Seguro - Bahia. 2009. Gestão do Trabalho e Educação na Saúde. Fundação Oswaldo Cruz, Bahia, 2009.

KRISHANAM, A et al. Evaluation of computerized health management information system for primary health care in rural India. BMC Health Services Research. v.10, n. 310, 2010.

KIVINEM, T; LAMMINTAKANEN, J. The success of a management information system in health care - a case study from Finland. International Journal of Medical Informatics. v.82, p.90-97, 2013.

KUMAR, $\mathrm{R}$ et al. The human resource information system: a rapid appraisal of Pakistan's capacity to employ the too. BMC Medical Informatics \& Decision Making. v.13, n.104, 2013.

KHAN, W. A et al. An adaptive semantic based mediation system for data interoperability among health information systems. J Med Syst. v.38, n.28, 2014.

LIMA, C. R. A et al. Review data quality dimensions and applied methods in the evaluation of health information systems. Caderno de Saúde Pública. Rio de Janeiro. v. 25, n. 10, p. 2095-2109, 2009.

LIMA, A. P; CORRÊA, A. C. P. Production of data for the pre-natal information system in basic health units. Revista de Enfermage. São Paulo, v. 47, n.4, p. 876-83, 2013.

LUDMER, M; LUDMER, G. Gestão integrada da auditoria médica e sistemas de informação: um estudo de caso em uma cooperativa de trabalho médico. Revista de Gestão em Sistemas de Saúde. São Paulo. v.2, n.1, p.116-139, 2013.

MARTINELI, R. M. F. Tecnologia da informação na construção do conhecimento: uma abordagem a partir do modelo de Nonaka \& Takeuchi. 2001. Dissertação (mestrado). Universidade Federal de Santa Catarina, Florianópolis, Santa Catarina, 2001.

MACEDO, C. P. C et al. Análise de dados do Sistema de Informação de Mortalidade (SIM) e Comitê de Mortalidade Materna (CMM) no Rio Grande do Norte. Revista Brasileira Promoção Saúde. v.25, n.4, p.413-419, 2012. 
MENDES, M. A. R. Redes sociais com base em dados provenientes de Sistemas de Informação para a Saúde. 2013. Dissertação (mestrado). 134 f. Universidade Aberta, Lisboa, 2013.

MEDEIROS, K. R et al. Bases de dados orçamentários e qualidade da informação: uma avaliação do Finanças do Brasil (Finbra) e do Sistema de Informação sobre Orçamentos Públicos em Saúde (Siops). Rev. Adm. Pública. v. 49, n. 5, p. 1113-1133, 2014.

MORAES, G. H; DUARTE, E. C. Análise da concordância dos dados de mortalidade por dengue em dois sistemas nacionais de informação em saúde, Brasil, 2000-2005. Caderno de Saúde Pública. v. 25, n. 11, p.2354-2364, 2009.

MEDEIROS, K. R et al. O sistema de informação em saúde como instrumento da política de recursos humanos: um mecanismo importante na detecção das necessidades da força de trabalho para o SUS. Ciência \& Saúde Coletiva. v.10, n.2, p. 433-440, 2005.

NYAMTEMA, A. S. Bridging the gaps in the health management information system in the context of a changing health sector. Medical Informatics \& Decision Marking. v. 10, n. 36, 2010.

OLIVEIRA, O. C. Sistema de informação da atenção básica: análise do processo de produção de dados e informações em equipe de saúde da família de Cuiabá-MT. 2010. Dissertação (mestrado). 165 f. Faculdade de Enfermagem, Universidade Federal do Mato Grosso, Mato Grosso, 2010.

OBSERVARH. Rede Observatório de Recursos Humanos em Saúde. Disponível em: <http://www.observarh.org.br/observarh/ repertorio/index.htm>. Acesso em: 13 out. 2015.

PATEL, S. V et al. Validation of the immunization data reported under health management information system by the Primary Health Centres of Rural Vadodara. Journal of Health Management. v.17, n.2, p.154-162, 2015.

PERES, S. V. Uso da técnica de linkage nos sistemas de informação em saúde: aplicação na base de dados do Registro de Câncer de base populacional no município de São Paulo. 2011.Tese (doudotado). Faculdade de Saúde Pública, Universidade de São Paulo, São Paulo, 2011.

PINTO, I. C et al. Os Sistemas de informação em atenção primária como instrumento de gestão em saúde: análise de experiências na Espanha. Caderno de Saúde Coletiva. v.18, n. 2, p.291-7, 2010.

PIRES, F. A. Ambiente para extração de informação epidemiológica a partir da mineração de dez anos de dados do Sistema Público de Saúde. 2011. 206 f. Tese (doutorado). Faculdade de Medicina, Universidade de São Paulo, São Paulo, 2011. 
PINHEIRO, A. L. S. O uso dos sistemas de informação como ferramenta para a tomada de decisão pela gestão da saúde em municípios do sul da Bahia. 2014. Tese (doutorado). Escola de Enfermagem de Ribeirão Preto, Universidade de São Paulo, São Paulo. 2014.

QAZI, M. S; ALI, M. Health management information system utilization in Pakistan: Challenges, pitfalls and the way forward. BioScience Trends. v.5, n. 6, p.245-254, 2011.

REEDER, B; TURNER, A. M. Scenario-based desing: A method for connecting information system design with public health operation and emergency management. v. 44, p.978-988, 2011.

RIBAS, C. E. Sistema de gestão de segurança da informação em organizações da área da saúde. 2010. 104 f. Dissertação (mestrado). Faculdade de Medicina de São Paulo, São Paulo. 2010.

RILEY, P. L et al. Information systems on human resources for health: a global review. Human Resources for Health. v.10, n.7, 2012.

RIPSA. Rede Interagencial de Informação para a Saúde. Biblioteca Virtual em Saúde. Disponível em: <http://www.ripsa.org.br/>. Acesso em: 13 out. 2015.

RODRIGUES, C. G et al. Os sistemas de informações em saúde: do processo de trabalho à geração dos dados em Minas Gerais. CEDEPLAR, 2008.

SANTOS, J. P. C. Gestão da mudança na implementação de sistemas de informação em organizações de saúde. Dissertação (mestrado). 90 f. Escola de Comunicação, Arquitectura, Artes e Tecnologias da Informação. Universidade Lusófona de Humanidades e Tecnologias, Lisboa, 2012.

SANTOS, S. R. S et al. Information system in health: management and support in the Brazilian unified health system. Cogitare Enferm. v. 19, n. 4, p. 772-9, 2014.

SILVEIRA, E. A. Indicadores de Saúde Infantil em Goiânia, Goiás, no período de 2000 a 2004, segundo dados do sistema de informação da atenção básica - SIAB. Revista Eletrônica de Enfermagem. v.09, n. 03, p.674-686, 2007.

SILVA, L. C. Modelo de decisão para alocação de recursos humanos em projetos de sistemas de informação. 2009. 79 f. Dissertação (Mestrado) - Universidade Federal de Pernambuco, Pernambuco, 2009.

SILVA, C. F; LEITE, A. J. M; ALMEIDA, N. M. G. S. Linkage between live birth and infant death records in a municipality in Northeast Brazil: quality of the information system. Caderno de Saúde Pública. Rio de Janeiro. v. 25, n. 7, p. 1552-1558, 2009. 
SILVA, H. O; CARVALHO, M. L; GONÇALVES. Tuberculosis/HIV coinfection in Brazilian state capitals: comments from the data of the Information System of Notifiable Diseases. RBPS. v. 22, n. 3, p. 172178, 2009.

SILVA, L. C; COSTA. A. P. C. S. Decision model for allocating human resources in information system projects. Project Management. v.31, p.100-108, 2013.

SOUZA, R. C; FREIRE, M. S; ALMEIDA, R. T. Sistema de Informação para integrar os dados da assistência oncológica ambulatorial do Sistema Único de Saúde. Caderno de Saúde Pública. v.26, n.6, p.1131-1140, 2010.

SPERO, J. C; MCQUIDE, P. A; MATTE, R. Tracking and monitoring the health workforce: a new human resources information system (HRIS) in Uganda. Human Resources for Health. v.9, n.6, 2011.

SUZUKI, K. M. F. O uso de método de relacionamento de dados (record linkage) para integração de informação em sistemas heterogêneos de saúde: estudo de aplicabilidade entre níveis primário e terciário. 2010. 121 f. Tese (doutorado). Faculdade de Medicina de Ribeirão Preto, Universidade de São Paulo, São Paulo. 2012.

THAINES, G. H. L. S et al. Produção, fluxo e análise de dados do sistema de informação em saúde: um caso exemplar. Texto Contexto Enfermagem. Florianópolis, v. 18, n. 3, p. 466-74, 2009.

WATERS, K. P et al. Kenya's health workforce information system: A model of impact on strategic human resources pality, planning and management. International Journa of Medical Informatics. v.82, p.895-902, 2013.

WAGENAAR, B. H et al. Effects of a health information system data quality intervention on concordance in Mozambique: time-series analyses from 2009-2012. Population Health Metrics. v.13, n.9, 2015.

WEIMEI, Z. Design of function and framework of enterprise human resource management information system. Information Technology Journal. v.12, n.8, p.1593-1599, 2013.

ZAFAR. H. Human resource information systems: information security concerns for organizations. Human Resource Management Review. v.23, p.105-113, 2013. 


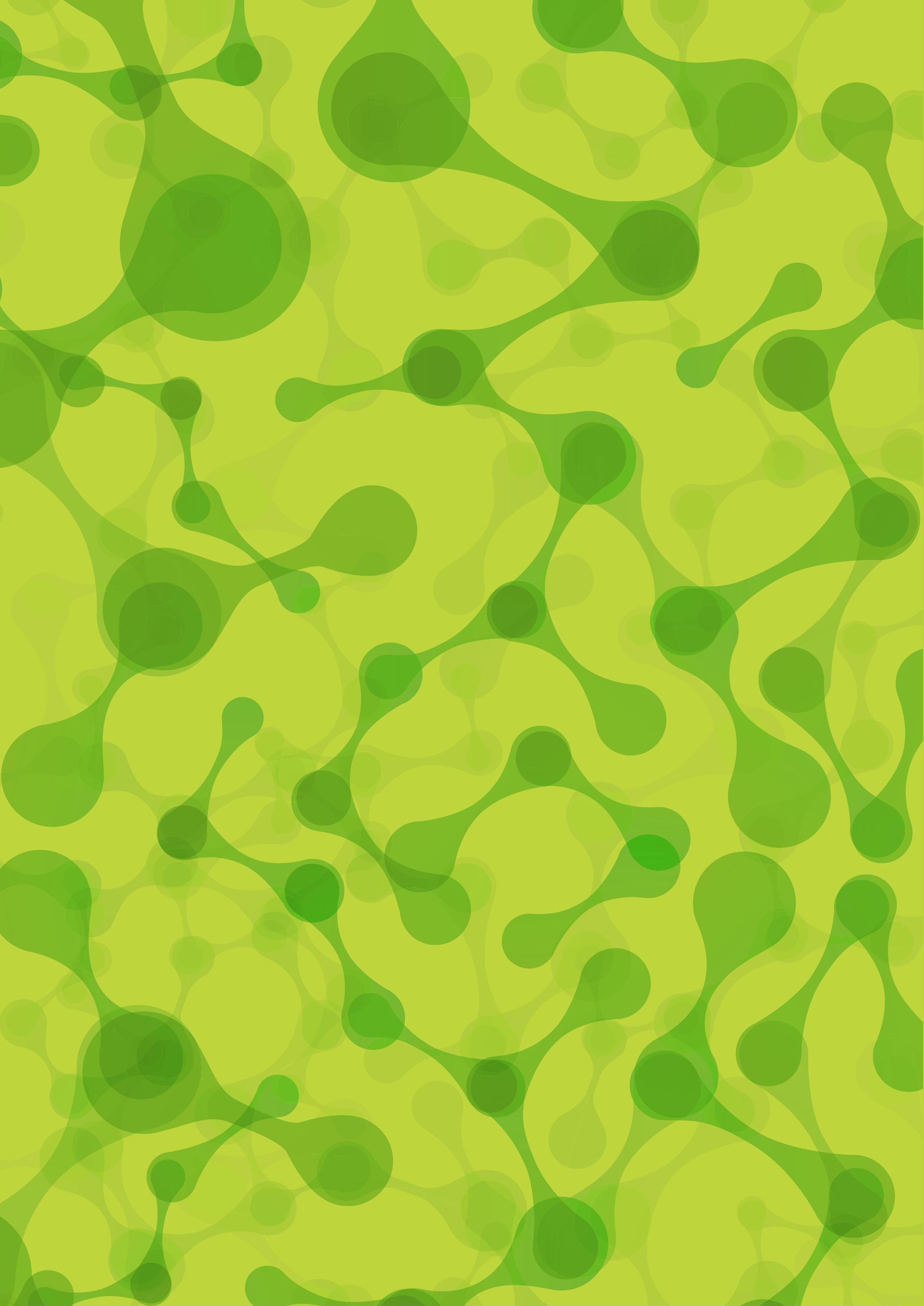

